\title{
Dynamics of confined plumes during short and ultrashort pulsed laser ablation of graphite
}

\author{
S. J. Henley,* J. D. Carey, and S. R. P. Silva \\ Nano-Electronics Centre, Advanced Technology Institute, School of Electronics and Physical Sciences, University of Surrey, Guildford, \\ GU2 7XH, United Kingdom \\ G. M. Fuge and M. N. R. Ashfold \\ School of Chemistry, University of Bristol, Bristol, BS8 1TS, United Kingdom \\ D. Anglos \\ Institute of Electronic Structure and Laser, Foundation for Research and Technology - Hellas, P.O. Box 1527, GR-711 10 Heraklion, \\ Greece
}

(Received 5 August 2005; revised manuscript received 22 September 2005; published 9 November 2005)

\begin{abstract}
The optical emission from electronically excited $\mathrm{C}$ species in the ablation plume following the short (ns) and ultrashort (fs) UV pulsed laser ablation of graphite is studied. Wavelength, time and spatially resolved imaging of the plume, in background pressures of inert gases such as argon and helium, is performed. Analysis of images of optical emission from $\mathrm{C}^{+^{*}}$ ions and $\mathrm{C}_{2}^{*}$ radicals, yielded estimates of the apparent velocity of emitting species, which appear to arise both from the initial ablation event and, in the presence of background gas, mainly from impact excitation. At elevated background pressures of argon $\left(P_{\mathrm{Ar}}\right)$, the formation and propagation of a shock wave is observed for ns pulses, whereas for fs pulses, the propagation of two shock waves is observed. During fs ablation, the first shock wave we associate with an initial burst of highly energetic/electronically excited ablated components, indicative of an enhanced fraction of non-thermal ejection mechanisms when compared with ns ablation. The second shock wave we associate with subsequently ejected, slower moving, material. Concurrent with the plume dynamics investigations, nanostructured amorphous carbon materials were deposited by collecting the ablated material. By varying $P_{\mathrm{Ar}}$ from 5 to $340 \mathrm{mTorr}$, the film morphology could be changed from mirror smooth, through a rough nanostructured phase and, at the highest background pressures for ns pulses, to a low density cluster-assembled material. The evident correlations between the film structure, the mean velocities of the emitting $\mathrm{C}$ species, and their respective dependences upon $P_{\mathrm{Ar}}$ are discussed for both pulse durations. In addition, we comment on the effect of observed initial plume dynamics on the subsequent $\mathrm{C}$ cluster formation in the expanding plume.
\end{abstract}

DOI: 10.1103/PhysRevB.72.205413

PACS number(s): 61.43.-j, 52.38.Mf, 81.15.Fg

\section{INTRODUCTION}

The presence of ambient gas can have a dramatic effect on the expansion dynamics of laser ablated plasmas, ${ }^{1}$ which in turn can affect the nature of the species present in the plume. Pulsed laser ablation ${ }^{2}$ (PLA) of graphite results in the deposition of quite different carbonaceous structures depending on the pressure of ambient gas in the chamber. ${ }^{3,4}$ In vacuum, PLA of graphite is typically used to produce smooth, hard, high density amorphous carbon $(a-C)$ films with a high $s p^{3}$ bonded $\mathrm{C}$ content (up to 90\%). ${ }^{5,6}$ The high $s p^{3}$ content has been attributed to local densification, caused by the impact of high energy $\mathrm{C}$ atoms/ions. This subplantation model, has been shown to provide a suitable representation of the growth mechanism for $a-\mathrm{C}$ films grown by a variety of techniques. ${ }^{7}$ The introduction of a low pressure of inert gas into the growth chamber reduces the kinetic energy of the growth species due to the increased collision frequency. In this regime, plume splitting into fast ( vacuum speed) and collision retarded components has been observed. ${ }^{1,8}$ At higher pressures plume confinement, and plume heating occurs, which leads to enhanced emission from the plume core and enhanced excitation/ionization at the plume front. High collision rates encourage conversion of the plume stream velocity into thermal energy. Compared with growth in vacuum, these conditions have been shown to yield lower density nanostructured amorphous carbon (NAC) films, ${ }^{4,9}$ which can exhibit a range of useful properties including low thresholds for electron field emission ${ }^{10}$ and high porosities. ${ }^{4}$ Recent work on high-repetion-rate laser ablation of graphite in high background pressures of argon has shown that these conditions are suitable for the deposition of carbon nanofoam. ${ }^{11}$ This cluster assembled filamentary material has been shown to demonstrate ferromagnetic behavior ${ }^{12}$ suggesting possible applications in spintronic devices.

Traditionally, PLA has been performed using lasers giving nanosecond (ns) duration pulses. However, the advent of subpicosecond [typically, and henceforth called femtosecond (fs)] pulsed lasers opened the possibility of decoupling the initial photoexcitation of the target material from the subsequent dynamics of the removed atomic, ionic, and molecular plume components. This decoupling removes a layer of complexity from the ablation process, thereby facilitating the investigation and understanding of PLA mechanisms. U1trashort pulsed lasers offer dramatically higher peak-powers than ns lasers and hence, the ablation mechanisms can be significantly different. Two different models are often proposed to describe the ablation by these ultrashort pulses. In the plasma-annealing model, the rapid rise in the number of electrons in excited states at the irradiated zone can produce 
bond softening by Coulomb repulsion allowing ultrafast melting. In this regime the plume of ablated material can contain ions ejected with "suprathermal" energies ${ }^{13-15}$ (i.e., emitted at velocities too large to be accounted for by thermal evaporation). In the two temperature model, ${ }^{16}$ the temperature of the electrons is treated separately to that of the lattice, the laser energy coupling only to the electrons. Thus, the laser essentially produces an immediate rise in the electron temperature. Hot electrons then transfer energy to the lattice (typically on a ps timescale, depending on the electronphonon coupling strength). The ablation is thus pictured as fast thermal evaporation.

In this paper we compare the dynamics of the ablation plume, during the $248 \mathrm{~nm}$ pulsed laser ablation of graphite, for pulses of ns and fs duration. The ablation is performed in background pressures of argon and helium $\left(P_{\mathrm{Ar}}\right.$ and $\left.P_{\mathrm{He}}\right)$. We investigate how the background pressure affects the kinetic energies of species in the ablation plume by using time-gated imaging methods ${ }^{17}$ to monitor the temporal and spatial evolution of the accompanying optical emission as a function of $P_{\text {Ar }}$. Particular attention is focussed on emission from electronically excited $\mathrm{C}^{+}$ions (henceforth $\mathrm{C}^{+*}$ ), which are considered to arise both from $\mathrm{C}^{2+}$ ion/electron recombination processes in the early stages of plume expansion and, at elevated background pressures, from collision induced impact ionization/excitation of $\mathrm{C}$. The apparent velocities of these emitting species are estimated, and correlations between these velocities and the structure of the resulting films explored.

\section{EXPERIMENTAL DETAILS}

The ablation plume imaging apparatus consists of a stainless steel vacuum chamber which was evacuated using a turbomolecular pump, backed by a rotary pump to a base pressure of $10^{-6}$ Torr. The chamber was equipped with several side arms, each of which was sealed with a quartz window, permitting wide spectral-range optical observation of the plume produced during ablation. The incident laser beam propagated through one of the side arms, in the $x y$ plane at $45^{\circ}$ to the target surface normal-here defined as the $x$ axis. The viewing port lay in the $x y$ plane also and allowed optical emission accompanying the ablation plume to be monitored by viewing along the $y$ axis (i.e., parallel to the target surface). The highly oriented pyrolytic graphite target (Kurt J. Lesker, $99.99 \%$ pure), was rotated during the experiments in order to avoid repeated ablation of the same spot on the target. Femtosecond $248 \mathrm{~nm}$ laser pulses were generated using a $\mathrm{XeCl}$ excimer pumped dye laser system producing 450 fs pulses from a distributed feedback dye laser at $496 \mathrm{~nm}$ which, after frequency doubling, were amplified in the $\mathrm{KrF}$ excimer laser cavity. ${ }^{18,19}$ The KrF cavity (Lambda-Physik EMG150MSC), operating unseeded, provided pulses of 15 ns full width at half maximum (FWHM) at $248 \mathrm{~nm}$. The outputs of the nanosecond excimer laser and the frequency doubled dye laser had markedly different spatial profiles, beam divergences, and peak powers, which meant that these two sources produced very different spot sizes when focused at the target. Typical laser pulse energies (and flu- ences) employed in the experiments described here were $\sim 110 \mathrm{~mJ} / \operatorname{pulse}\left(6 \mathrm{~J} / \mathrm{cm}^{2}\right)$ in the case of ns ablation and $\sim 13 \mathrm{~mJ} /$ pulse $\left(6 \mathrm{~J} / \mathrm{cm}^{2}\right)$ for the fs studies. Although the fluences used were similar, the power density was nearly 5 orders of magnitude lower for the ns, as compared with the fs, pulses.

The plume emission was collected in two different ways. Spatially resolved, wavelength dispersed spectra were recorded by focusing (in a 1:1 magnification configuration) the emission onto a quartz fiber, directed so as to view a localized column along the $y$ axis. The exit of the fiber abutted the entrance slit (width set at $30 \mu \mathrm{m}$ ) of a $0.32 \mathrm{~m}$ spectrograph (TRIAX-320, Jobin Yvon/Spex) equipped with three, user selectable, holographic gratings, with 600,1800 , and 2400 lines $/ \mathrm{mm}$, providing spectral resolutions of $0.4,0.15$, and $0.1 \mathrm{~nm}$, respectively. The resulting dispersed emission spectrum was recorded with an intensified charge coupled device ( $i$-CCD DH520-18F, Andor Technology), the sensitivity of which spanned the wavelength range $350-900 \mathrm{~nm}$. The spectra were collected using a gate time of $1 \mu \mathrm{s}$, which opened $120 \mathrm{~ns}$ after the laser pulse. This delay time was the minimum response time of the electronics used. By translating the position of the entrance of the fiber, the $x$ dependence of the optical emission spectrum could be measured, with a spatial resolution of $\sim 0.6 \mathrm{~mm}$. A second means of investigating the plume emission involved imaging the evolving spatial distributions of selected emitting species using the $i$-CCD camera in time-gated detection mode, decoupling it from the spectrometer and focusing the optical emission (either total emission, or just the fraction transmitted through a suitably chosen narrow bandpass interference filter) onto the $1024 \times 256$ diode array. The spatial calibration of the $i$-CCD image was achieved by taking an image of a ruler positioned in the $x z$ plane and illuminated by a white light source. The minimum time delay at which an i-CCD image of the plume could be taken was limited to $t=120 \mathrm{~ns}$ by the response time of the available electronics. Optical emission spectra and wavelength filtered plume images were obtained for $P_{\mathrm{Ar}}$ values in the range $0-340$ mTorr and at $P_{\mathrm{He}}=340 \mathrm{mTorr}$ to 4 Torr.

Films were deposited by ns PLA, of the same graphite target, in a separate ablation chamber using a LambdaPhysik LPX 210i excimer laser operating at $248 \mathrm{~nm}$, with a pulse duration of $25 \mathrm{~ns}$. The $60 \mathrm{~mJ}$ laser pulse was focused onto the rotating target, producing an incident fluence of $\sim 6 \mathrm{~J} / \mathrm{cm}^{2}$. Here the same fluence was used for the ns deposition and plume analysis experiments. However, as the laser spot sizes were different, there is likely to be slightly different angular distributions of material for the two deposition systems. Thus, to allow fair comparison, optical emission spectroscopy, microscopic analysis of deposits, and velocity analysis of the plume expansion were performed only along the target surface normal to minimize any discrepancy. The growth chamber was evacuated using a turbo molecular pump to $9 \times 10^{-8}$ Torr, then back-filled with argon to the required pressure. The material ejected during fs PLA was collected in the same vacuum chamber used for the imaging studies, concurrent with the plume dynamics experiments. A larger number of laser shots was required for fs pulses, to produce a similar film thickness as for ns ablation, as it ap- 


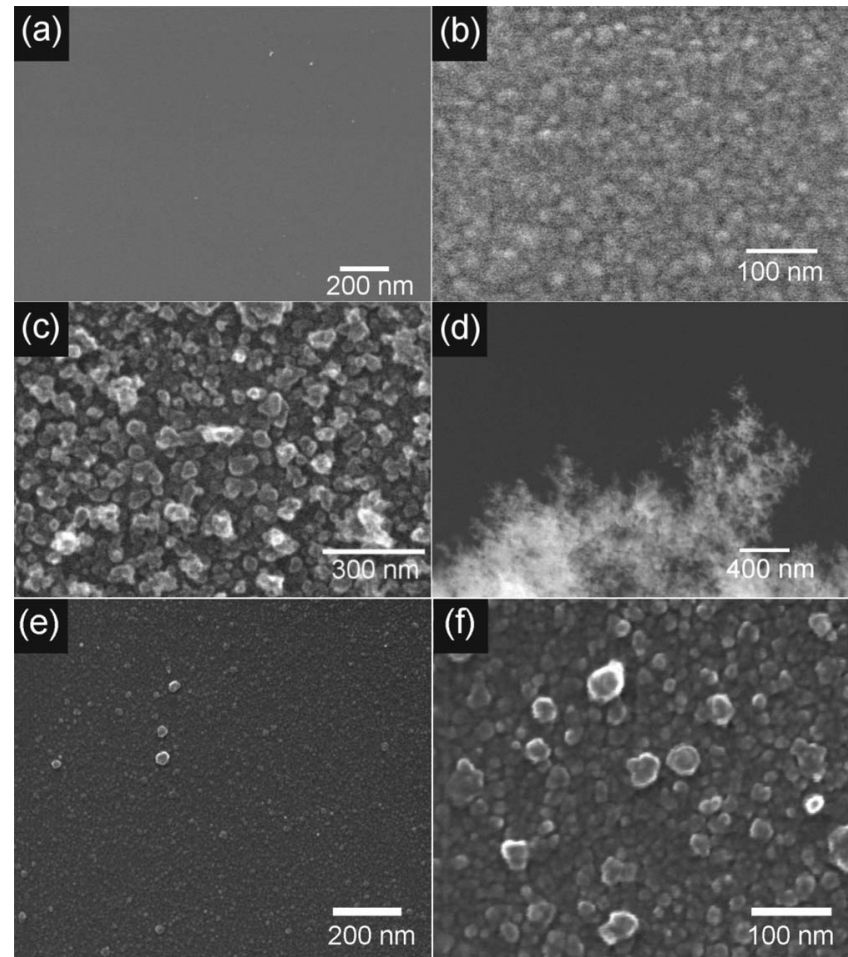

FIG. 1. SEM images of a range of samples deposited with (a)(d) ns pulses and (e)-(f) with fs pulses. During deposition $P_{\text {Ar }}$ was maintained at (a) 5 mTorr, (b) 40 mTorr, (c) 100 mTorr, (d) 340 mTorr-sample shown in cross section to emphasize filamentary nature, (e) 340 mTorr, and (f) 500 mTorr.

peared that less material is ablated per shot. For both growth setups, films were deposited onto Si substrates at various $P_{\mathrm{Ar}}$ ranging from 5 to $500 \mathrm{mTorr}$. The target to substrate distance was fixed at $6 \mathrm{~cm}$. The deposition rate was found to be $P_{\mathrm{Ar}}$ dependent, and the number of laser shots was thus varied (in the range 2000-10 000) in order that the final film thickness was within a fixed range. The surface morphology of the films was investigated in a Hitachi S4000 field emission gun scanning electron microscope (SEM) operating at $20 \mathrm{kV}$. The nanostructure of the films was investigated using a Philips CM200 high-resolution transmission electron microscope (TEM). Laser Raman spectroscopy (Renishaw Ramanscope, $514.5 \mathrm{~nm}$ excitation) was used to investigate the structure and bonding configurations in the films.

\section{RESULTS AND DISCUSSION}

\section{A. Analysis of deposited material}

For ns pulses, the surface morphology of the deposited material was examined by SEM. Figure 1 shows SEM images of a range of samples deposited at different $P_{\mathrm{Ar}}$. We find that films deposited at the lowest $P_{\mathrm{Ar}}$ used (5 mTorr) are essentially indistinguishable from tetrahedral amorphous carbon (ta-C) films grown in vacuum, being hard, scratch resistant and having a "mirror" smooth morphology [see Fig. 1(a)]. Increasing $P_{\text {Ar }}$ to 40 mTorr [Fig. 1(b)] yielded films that are much rougher. The SEM images reveal nanoscale roughness, with an estimated scale of $d \sim 15 \pm 3 \mathrm{~nm}$. Larger

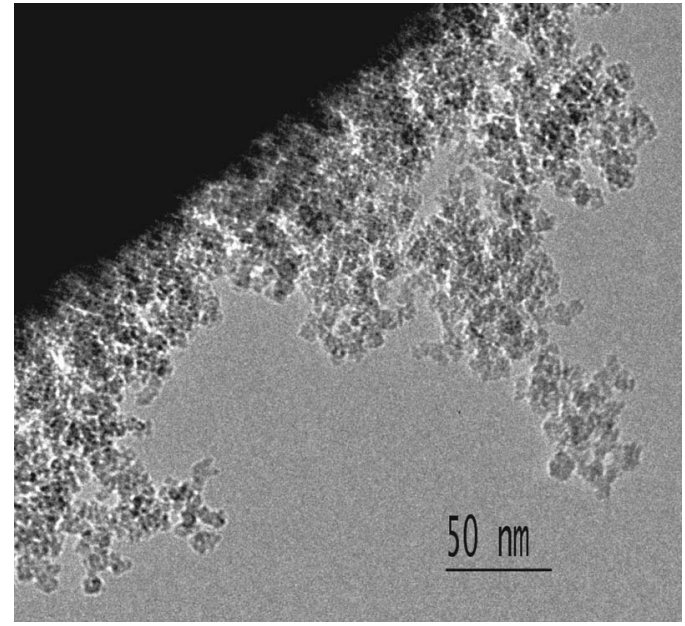

FIG. 2. High-resolution TEM image of the nanostructure of the sample deposited at $P_{\mathrm{Ar}}=340 \mathrm{mTorr}$.

thickness fluctuations $(d \sim 45 \mathrm{~nm})$ are clearly evident when $P_{\text {Ar }}$ was raised further, to 100 mTorr. These deposits could be easily scratched off with a pair of tweezers, consistent with the expected reduction in film hardness. Films grown at $P_{\text {Ar }}=340$ mTorr exhibited yet another morphology, wherein the nanoscale roughness was replaced by more filamentary growth, similar to the carbon nano-foam deposited by Rode et $a{ }^{11}$ These films, which could be removed simply by rubbing the surface, were deduced to have the lowest hardness and density of all of the $a$-C films grown in the present work. A high-resolution TEM image of this filamentary material is shown in Fig. 2. The nanostructure is seen to consist of nanoscale clusters with diameters of $\sim 10 \mathrm{~nm}$. These clusters are assembled in a fractal-like arrangement suggesting that the growth mechanism is characterized by diffusion limited aggregation. ${ }^{20}$

Figures 1(e) and 1(f) show SEM images of the material deposited by fs pulses at $P_{\mathrm{Ar}}=340$ and 500 mTorr, respectively. Nanoscale roughness is observed again, but the films appear smoother than those deposited at the same pressure using ns pulses. Even at the highest pressure, 500 mTorr, there was no evidence of the deposition of carbon-nanofoamlike material found for ns ablation. These observations are discussed in more detail later. As the fs-ablated material evidenced nanostructures comparable to material deposited by ns pulses at lower pressures, the fs plume dynamics studies presented later will concentrate mainly on higher pressures. As two different chambers were used during the deposition experiments, one possible source of error here is the accuracies of the two pressure gauges used. The ns and fs plume dynamics experiments, presented later, were all performed in the same chamber and thus will be internally consistent. For the ns growth chamber, however, no direct comparison was possible with the set pressure during the fs depositions. However, comparable nanostructures between the fs and ns deposits were only observed for a factor of 5 difference in pressure [compare Figs. 1(c) and 1(f)], which should be significantly outside the bounds of experimental uncertainty.

The density and bonding configuration of $a$-C films produced by PLA are determined primarily by the kinetic 
energy (and thus velocity) of the growth species as they impact onto the substrate. ${ }^{21}$ In particular, the flux of highly energetic $(\sim 100 \mathrm{eV}) \mathrm{C}$ species (ions and/or neutrals) is considered to be important for the formation of $a-\mathrm{C}$ films with a high $s p^{3}$ bonding fraction. It was thus logical to investigate how changing $P_{\mathrm{Ar}}$ affected the bonding in the as-deposited films. The Raman spectrum for each of the samples deposited, by ns ablation, was measured using $514.5 \mathrm{~nm}$ excitation. These results are presented elsewhere, ${ }^{22}$ but we present a summary here: Visible Raman spectra of $a$-C films are traditionally characterized as a combination of two bands, labeled the D and $\mathrm{G}$ peaks and attributed to the breathing mode of aromatic rings and in-plane bond stretching of pairs of $s p^{2}$ bonded C atoms, respectively. ${ }^{23-25}$ For $514.5 \mathrm{~nm}$ excitation the $\mathrm{D}$ and $\mathrm{G}$ peaks are typically centered at $\sim 1335 \mathrm{~cm}^{-1}$ and in the range $1580 \pm 20 \mathrm{~cm}^{-1}$, respectively. For films deposited at low $P_{\mathrm{Ar}}$ (below $\sim 15$ mTorr) we find that the complete Raman line shape can be fitted ${ }^{24}$ without any D peak contribution. This is characteristic of so-called tetrahedral amorphous carbon $(t a-\mathrm{C})$ films, for which the ratio of the $\mathrm{D}$ and $\mathrm{G}$ peak intensities $\left(I_{\mathrm{D}} / I_{\mathrm{G}}\right)$ tends towards zero as the fraction of $s p^{3}$ bonded $\mathrm{C}$ increases, and implies that the present films grown at $P_{\mathrm{Ar}}$ less than $\sim 15 \mathrm{mTorr}$ contain few if any six-membered $s p^{2}$ clusters.

Fitting the Raman line shapes of films deposited at higher $P_{\text {Ar }}$ required inclusion of progressively larger D peak contributions, implying an increase in ordering of the $s p^{2}$ phase due to the formation of larger clusters of aromatic rings. ${ }^{24}$ This increase in $\left(I_{\mathrm{D}} / I_{\mathrm{G}}\right)$ ratio also suggests an increase in the fraction of $s p^{2}$ bonded $\mathrm{C}$ in the films. ${ }^{22}$ These observations are similar to those of Siegal et al. ${ }^{4}$ who found little change in the Raman spectra of NAC films grown by PLA as $P_{\mathrm{Ar}}$ was increased through the range 175-300 mTorr. We also note that, though the density of these films is low, their Raman spectra are similar to those of high density amorphous carbon $(a-C)$, unlike the films deposited by cluster assembly. ${ }^{26}$

\section{B. Wavelength dispersed optical emission spectroscopy}

In order to explore the effect of background pressures of inert gas and the pulse duration on the film structure further, the dynamics of the plume of ablated material was studied by monitoring the temporal and spatial evolution of its optical emission. Initially wavelength dispersed optical emission spectra (OES) were measured at different $P_{\mathrm{Ar}}$, close to the ablation volume $(x \sim 0)$ and at $x \sim 5 \mathrm{~mm}$. Figure 3 shows such spectra, recorded in vacuum and at $P_{\mathrm{Ar}}=154$ mTorr, for ablation with ns pulses. The spectra exhibit numerous sharp lines all of which are attributable to emission from electronically excited $\mathrm{C}^{+*}$ ions and $\mathrm{C}^{*}$ and excited $\mathrm{Ar}$ ions and neutrals, as well as the characteristic Swan bands associated with excited $\mathrm{C}_{2}(d)$ radicals. A detailed list of these lines, assigning the emitter and emitting transition, is presented by Claeyssens et $a l .{ }^{17}$ The relative intensities, however, of these various emissions varies markedly between the spectra. $C_{2}^{*}$ emission is evident in the immediate ablation zone at all $P_{\mathrm{Ar}}$, but only at higher $P_{\mathrm{Ar}}$ does it persist to larger $x$, suggesting a role for collision induced formation of, and/or excitation to, $\mathrm{C}_{2}^{*}$.

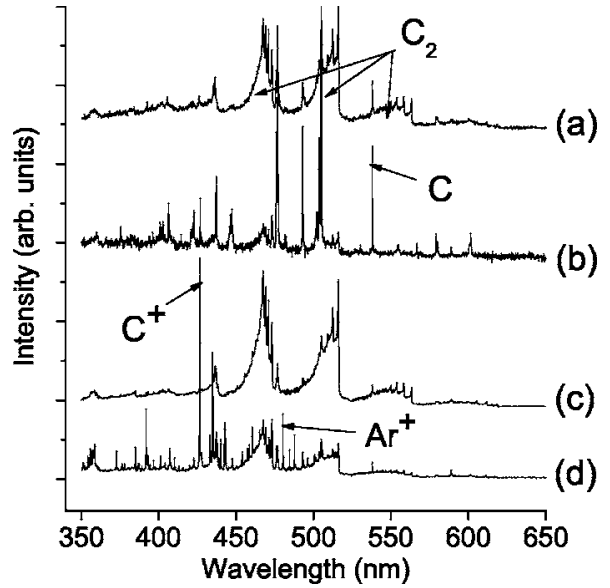

FIG. 3. Spatially resolved OES spectra of the plume, for ns ablation, measured at different $P_{\mathrm{Ar}}$ and distance $x$ from the ablation spot. (a) Vacuum and $x=0 \mathrm{~mm}$, (b) vacuum and $x=5 \mathrm{~mm}$, (c) $P_{\mathrm{Ar}}=154$ mTorr and $x=0 \mathrm{~mm}$, (d) $P_{\mathrm{Ar}}=154$ mTorr and $x=5 \mathrm{~mm}$.

We note that, even under vacuum, $\mathrm{C}_{2}^{*}$ emission makes a far greater relative contribution to the total OES than in the case of PLA of graphite at $193 \mathrm{~nm} .{ }^{27}$ The $\mathrm{C}^{+*}$ and $\mathrm{C}^{*}$ emissions are associated with transitions between excited states of the respective species. Radiation trapping is thus unlikely to be an issue, and the observed persistence of such emissions from plumes arising in PLA processes, in vacuum (over timescales that correspond to many natural radiative lifetimes) has been rationalized in terms of electron-ion recombination (EIR), leading to formation of highly excited (Rydberg) atoms and subsequent radiative cascade. ${ }^{27}$ Such a sequence, finally observed in the optical emission as a peak at 426.7 $\mathrm{nm}$, follows:

$$
\mathrm{C}^{2+}+e \rightarrow \mathrm{C}^{+* *}(\text { high } n, l, m) \rightarrow \rightarrow \rightarrow \mathrm{C}^{+*}(4 f) \rightarrow \mathrm{C}^{+*}(3 d) \text {. }
$$

In the case of ns and fs PLA in vacuum, therefore, $\mathrm{C}^{+*}$ emission is regarded as a signature of $\mathrm{C}^{2+}$ species, etc. In the present work, both $\mathrm{C}^{+*}, \mathrm{Ar}^{*}$ and $\mathrm{Ar}^{+*}$ emissions are seen to gain in relative intensity as $P_{\mathrm{Ar}}$ and $x$ increase (see Fig. 3). The latter emissions, in particular, provide clear indications of the involvement of one or more collision induced, postablation excitation routes.

After examining the ablation with ns pulses, we turn our attention to the OES produced by pulses of fs duration. Figure 4 shows such spectra, recorded in vacuum and at $P_{\mathrm{Ar}}=154 \mathrm{mTorr}$, for ablation with fs pulses. Again, two spectra are shown for each pressure, one taken from close to the ablation volume $(x \sim 0)$ [Figs. 4(a) and 4(c)] and one at $x \sim 5 \mathrm{~mm}$ [Figs. 4(b) and 4(d)] along the target surface normal. By comparing the spectra in Figs. 3 and 4, we observe that, for ablation in vacuum, the OES spectra at the ablation volume are very similar for fs and ns pulses. Both spectra are dominated by the $\mathrm{C}_{2}^{*}$ Swan band emission. This dominance of $\mathrm{C}_{2}^{*}$ emission could be a consequence of the detection method. The minimum delay time after the laser pulse that could be used for the start of the detection gate, was $120 \mathrm{~ns}$. It is likely that even after this delay a significant fraction of 


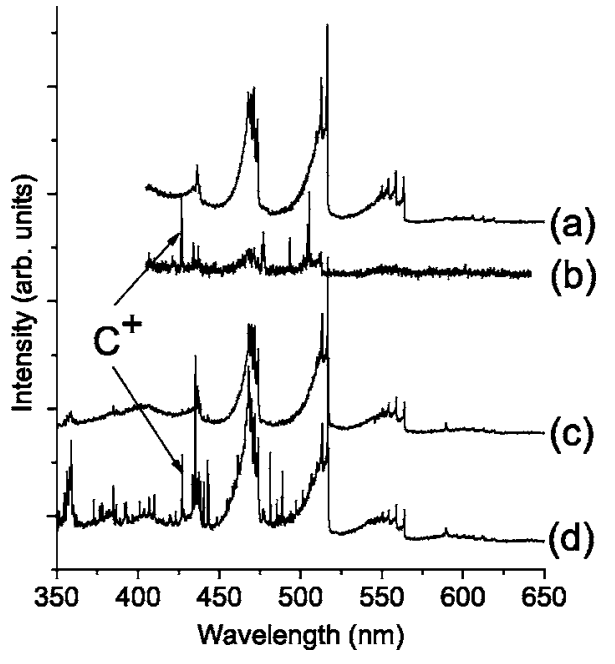

FIG. 4. Spatially resolved OES spectra of the plume, for fs ablation, measured at different $P_{\mathrm{Ar}}$ and distance $x$ from the ablation spot. (a) Vacuum and $x=0 \mathrm{~mm}$, (b) vacuum and $x=5 \mathrm{~mm}$, (c) $P_{\mathrm{Ar}}=154$ mTorr and $x=0 \mathrm{~mm}$, (d) $P_{\mathrm{Ar}}=154 \mathrm{mTorr}$ and $x=5 \mathrm{~mm}$.

the emitting $\mathrm{C}$ atoms and ions will have escaped past the detection column, and indeed these atomic and ionic emissions appear when the detection zone is moved to $x=5 \mathrm{~mm}$. However, for ns ablation [see spectrum (a) in Fig. 3], C* emission is still detected at the ablation spot indicating that there are significant differences in the ejection mechanisms between the two pulse durations. This will be discussed in more detail later.

The $\mathrm{C}_{2}^{*}$ emission is localized at the spot for fs ablation in vacuum and, similar to ns ablation, only extends further away from the target at elevated background pressures. Similar observations have been made for the ablation of graphite in $\mathrm{N}_{2} \cdot{ }^{28}$ At $5 \mathrm{~mm}$ from the target, for ablation in vacuum, the most intense emission comes from the $\mathrm{C}^{+}$ions for fs ablation, instead of from excited $\mathrm{C}$ atoms as was the case for ns ablation. This is consistent with the observation of an enhanced degree of plume ionization for ultrashort pulsed ablation when compared with using nanosecond pulses, ${ }^{17,29}$ although no information can be obtained on the distribution of ground state material using optical emission measurements. For the fs ablation at elevated pressures we observe that the enhancement, compared to ablation in vacuum, of the $\mathrm{C}^{+*}$ emission is not as large as for ns pulses. The spectrum at $x=5 \mathrm{~mm}$ is still dominated by the $\mathrm{C}_{2}^{*}$ emission, which also increases in intensity slightly compared to the signal at $x=0 \mathrm{~mm}$. This either indicates an increased production of $\mathrm{C}_{2}$ fragments during the initial ablation, compared with ns pulse ablation, or more collision induced formation ${ }^{30}$ of, and/or excitation to, $C_{2}^{*}$. Simulations of the fs ablation of graphite ${ }^{14}$ have shown that ultrafast pulses produce significant carbon chains and clusters in addition to fast monomers, so it is likely that the enhanced $C_{2}^{*}$ signal for fs pulses indicates an increased production of $\mathrm{C}$ clusters by fs pulses. Another factor affecting the relative numbers of $\mathrm{C}$ clusters in the plumes, produced by ns and fs pulses, is the interaction of the laser with the ablation plume. For fs pulses the laser pulse has ended before the plume has formed, so no interaction is pos-

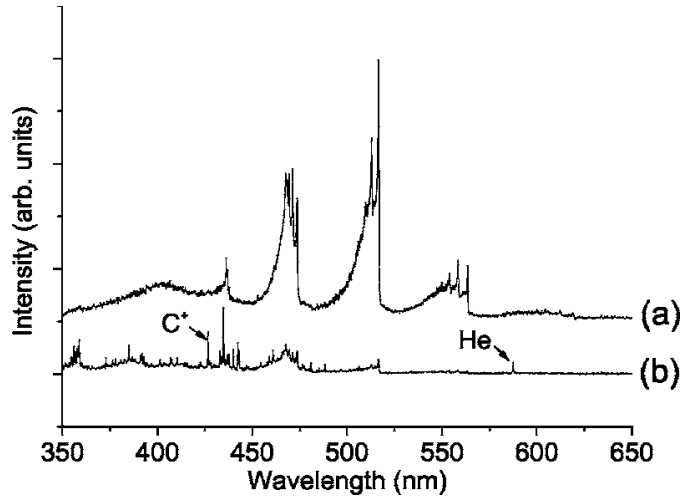

FIG. 5. Spatially resolved OES spectra of the plume, for fs ablation in 340 mTorr of helium, measured at different distances $x$ from the ablation spot. (a) $x=0 \mathrm{~mm}$ and (b) $x=5 \mathrm{~mm}$.

sible. For ns pulses however, the laser can interact with the ablated species. As the laser photon energy is large enough to break $\mathrm{C}-\mathrm{C}$ bonds, photodissociation of molecular $\mathrm{C}_{n}$ species is possible. This effect is expected to be enhanced for ablation at $193 \mathrm{~nm}$, and indeed, Claeyssens et al. observed no emission from $\mathrm{C}_{2}^{*}$ for ablation at this wavelength. ${ }^{27}$

In addition to ablation in Ar, OES spectra were measured for the fs ablation of graphite in background pressures of He. Figure 5 shows spatially resolved OES spectra of the plume, for fs ablation in $340 \mathrm{mTorr}$ of He, measured at $x \sim 0 \mathrm{~mm}$ and $x \sim 5 \mathrm{~mm}$ from the target. At the ablation spot the spectrum is again dominated by the $\mathrm{C}_{2}^{*}$ emission. Away from the ablation spot, the emission from the $\mathrm{C}_{2}^{*}$ Swan band system does not retain its intensity as was observed for ablation at lower pressures of $\mathrm{Ar}$, and only one line attributable to $\mathrm{He}^{*}$ is observed. This is likely due to the smaller size, and hence smaller collisional cross section, of He atoms compared to Ar. Thus, a much higher pressure of $\mathrm{He}$ will be required to cause significant impact excitation of the ablated species. We will return to discuss possible collisional excitation and impact ionization schemes (for both $\mathrm{C}^{+*}$ and $\mathrm{C}_{2}^{*}$ ) later. Also visible in the spectra taken at $x=0 \mathrm{~mm}$ is a broad emission band in the range $360-450 \mathrm{~nm}$, which has been attributed to emission from $\mathrm{C}_{3}^{*}$ radicals. ${ }^{31}$ This emission band is also observed at the focal spot for the fs ablation in vacuum and $\mathrm{Ar}$ (see Fig. 4), perhaps indicating again that more small clusters are ablated for fs pulses, compared with ns ablation.

Before considering the additional insights provided by time-gated imaging measurements, we summarize the conclusions drawn from the OES produced by ns and fs pulses at $248 \mathrm{~nm}$.

(i) fs pulses, incident on a graphite target in vacuum, directly ablate a larger fraction of material in highly excited, ionized states. For ns pulses the bulk of the plasma is formed by laser-plume interactions.

(ii) The $\mathrm{C}_{2}^{*}$ Swan band emission appears as the dominant emission at the focal spot, for both pulse durations, regardless of the pressure of ambient gas.

(iii) In plumes confined by the presence of background inert gas, the majority of the $\mathrm{C}^{+*}$ emission is expected to come from impact ionization of $\mathrm{C}$ atoms and is a more significant component of the spectra for ns pulses, compared to fs. 


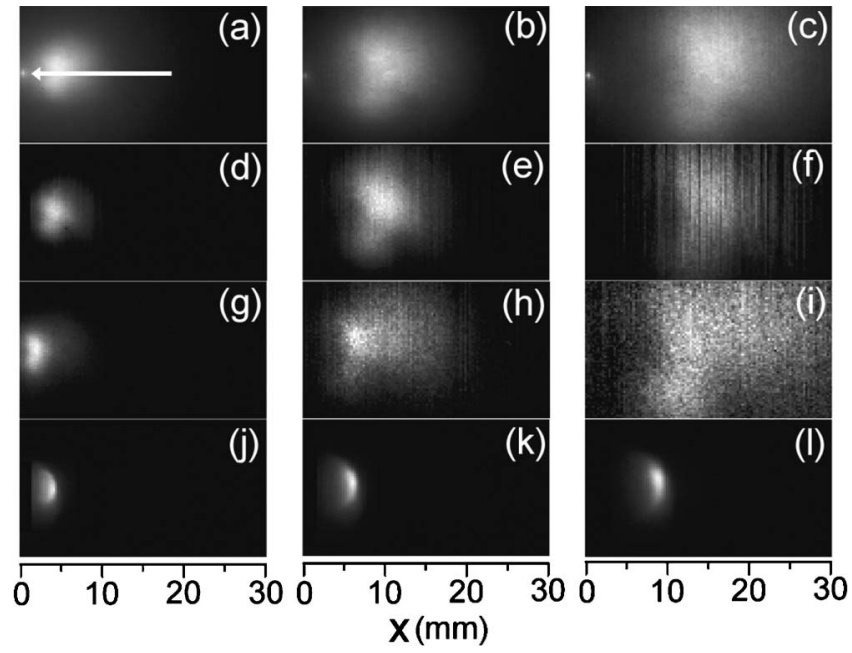

FIG. 6. Wavelength filtered CCD images of the ns ablation plume for different values of $P_{\mathrm{Ar}}=$ (a)-(f) vacuum, (g)-(i) 20 mTorr, and (j)-(l) 154 mTorr. Images (a)-(c) are unfiltered images, whereas the remainder were taken using a $426 \mathrm{~nm}$ narrow band pass filter to show only the $\mathrm{C}^{+*}$ emission at $426.7 \mathrm{~nm}$. All images were taken with a 20 ns gate but with a variable delay after the laser pulse of $120 \mathrm{~ns}$ for images in the first column, $250 \mathrm{~ns}$ for the second column and $400 \mathrm{~ns}$ for the third column. The white arrow in (a) indicates the axis of laser irradiation.

(iv) In confined plumes, the $\mathrm{C}_{2}^{*}$ emission is no longer confined to the focal spot and is a more significant component of the spectra for fs pulses, compared to ns. This could indicate that a larger fraction of the material is ablated as carbon clusters for fs pulses when compared with ns and/or that photodissociation of molecular $\mathrm{C}_{n}$ species, by the laser, is a significant effect for ns pulses.

(v) A significantly higher pressure of $\mathrm{He}$, compared to $\mathrm{Ar}$, is required to produce the same degree of plume confinement due the smaller collision cross section for $\mathrm{He}$.

\section{Time resolved imaging of plume emissions from specific species}

Figure 6 shows a series of time-gated CCD images of emission from the ns ablation plume for different values of $P_{\mathrm{Ar}}$. Images (a)-(f) are for PLA in vacuum, while (g)-(i) were recorded at $P_{\mathrm{Ar}}=20 \mathrm{mTorr}$, and (j)-(1) at $154 \mathrm{mTorr}$. Images (a)-(c) are of the total detectable emission, whereas all of the other images were recorded while viewing through a $426 \mathrm{~nm}$ narrow band pass filter chosen so as to transmit the $\mathrm{C}^{+*}\left(4 f ;{ }^{2} F^{\circ} \rightarrow 3 d ;{ }^{2} D\right)^{32}$ transition at $426.7 \mathrm{~nm}$. This is the most intense of all of the lines evident in Fig. 3(d). We recognize that this filter will also transmit emission from the weak Ar II line at $427.7 \mathrm{~nm}$, but this is not a major contributor to the emission spectra. All images were recorded using a 20 ns time gate on the intensifier, but with different time delays after the laser pulse: $t=120 \mathrm{~ns}$ for images in the first column, $250 \mathrm{~ns}$ (second column), and $400 \mathrm{~ns}$ (third column). The white arrow in (a) indicates the axis of laser irradiation. Each image has been averaged over multiple laser shots and scaled to allow use of the entire grey-scale range. By aver-

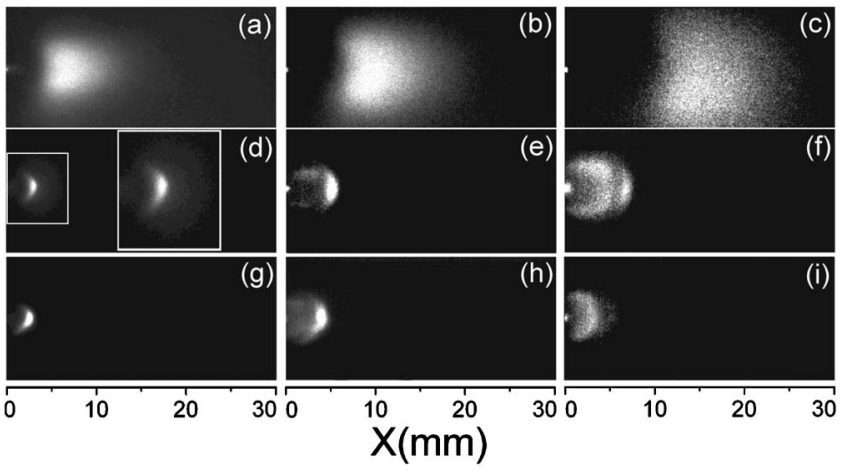

FIG. 7. Series of time-gated CCD images of emission from the fs ablation plume of graphite in different values of $P_{\mathrm{Ar}}$. All the images were taken using a filter that passes the $\mathrm{C}^{+*}$ emission at $426.7 \mathrm{~nm}$. (a)-(c) are for ablation in vacuum and are taken at delays of 120, 180, and $300 \mathrm{~ns}$ after the laser pulse, respectively. (d)-(f) show images obtained at $P_{\mathrm{Ar}}=154$ mTorr for delays of 120, 420, and $900 \mathrm{~ns}$ after the laser pulse. In (d) a magnified image of the shock front area is also displayed. (g)-(i) Are images obtained at $P_{\mathrm{Ar}}=340$ mTorr for delays of 120,420 , and $900 \mathrm{~ns}$, respectively.

aging over multiple shots very reproducible plume shapes could be obtained, reducing the effect of any shot-to-shot variation.

Comparing the images in the first and second rows of Fig. 6 , we see that the distribution of the total OES signal accompanying $248 \mathrm{~nm}$ PLA of graphite in vacuum is similar to that of the $\mathrm{C}^{+*}$ emission, though the unfiltered distribution appears somewhat broader (presumably because it is sampling a variety of species which need not all have the same velocity distributions). Clearly, the distribution of the $\mathrm{C}^{+*}$ emission is changed by the presence of background Ar. The peak in the $\mathrm{C}^{+*}$ emission images recorded at $P_{\mathrm{Ar}}=20$ mTorr of argon is retarded relative to the corresponding images in vacuum [see Figs. 6(g)-6(i)]. There are also hints of bimodality in the distribution along the $x$ axis, with a faster front component and a slower curved rear component [see, for example, Fig. 6(h)]. These trends become ever more pronounced with increasing $P_{\text {Ar }}$ [Figs. 6(j)-6(1)]. The distribution of $\mathrm{C}^{+*}$ emission appears as an arc, more characteristic of shock wave propagation. ${ }^{33}$ Thus, the peak in the spatial distribution of $\mathrm{C}^{+*}$ ions at high $P_{\mathrm{Ar}}$ is likely to be near the position of highest plume density and thus highest collisional excitation/ionization probability. In this case the majority of the $\mathrm{C}^{+*}$ is expected to come from impact ionised $\mathrm{C}$ atoms rather than from EIR of $\mathrm{C}^{2+}$. Also the peak of the early time distribution actually appears more advanced than in the corresponding $P_{\mathrm{Ar}}=20 \mathrm{mTorr}$ image. At longer delays after the laser pulses than presented here, the emission intensity from the shock front decays away and the spatial distribution along the $x$-axis broadens as the shock front dissipates into the background gas.

In addition to monitoring the plume emissions for ns pulses, the plume dynamics during fs ablation were also investigated. Figure 7 shows a series of time-gated CCD images of emission from the fs ablation plume of graphite in different values of $P_{\mathrm{Ar}}$. All the images in this figure were taken using the filter that passes the $\mathrm{C}^{+*}$ emission at 426.7 
nm. Figures 7(a)-7(c) are for fs ablation in vacuum and are taken at delays of 120, 180, and $300 \mathrm{~ns}$ after the laser pulse, respectively. Similar to ablation with ns pulses, the $\mathrm{C}^{+*}$ emission in vacuum appears as a broad distribution moving rapidly away from the ablation spot. When a background pressure of $\mathrm{Ar}$ is introduced the plume dynamics are again dramatically affected. Figures 7(d)-7(f) show images obtained at $P_{\mathrm{Ar}}=154 \mathrm{mTorr}$ for delays of 120,420 , and $900 \mathrm{~ns}$ after the laser pulse. Initially [Fig. 7(d)], the formation of a propagating shock wave is observed, similar to that observed with ns pulses. However, the shock wave is more confined along the $x$ axis and is more "arrow head" shaped, rather than presenting a smooth arc. Such "arrow head" shaped shock fronts are often observed for projectiles, such as bullets, traveling at supersonic velocities in air. This last observation suggests that the angular divergence of the ablated material is smaller for fs ablation when compared to ns pulses. Such a tightly forward-pointing distribution of ejected material is often observed for ablation with fs pulses. ${ }^{34}$ At later times the appearance of the $\mathrm{C}^{+*}$ emission distribution deviates from that observed with ns pulses. At $t=420 \mathrm{~ns}$ [Fig. 7(e)] two regions of emission are observed, oriented along the $x$ axis, at the sides of the plume. This observation could be explained by the formation of a region of lower pressure behind the shock front. Only at the edges of this region would material ejected at later times (or with slower initial velocities) come into contact with the background gas. The emission images are projections into $2 \mathrm{D}$ of the emission at the edges of an expanding region, thus the intensity received is greatest when viewing along a tangent to this region. By $900 \mathrm{~ns}$, the distribution changes to two arcs of emission [see Fig. 7(f)] both propagating forward, the arc closer to the target being broader than the more advanced one. This double shock wave may be evidence for the existence of (at least) two components in the fs ablation plume with very different velocity distributions. This conclusion is supported by recent measurements of the plume dynamics, in vacuum, after fs infrared ablation of graphite, where plumes with multi-component velocity structures were also observed ${ }^{35,36}$ It is suggested that the first component is associated with an initial burst of highly energetic/electrically excited ablated components, indicative of the enhanced fraction of nonthermal ejection mechanisms compared to ns ablation, and the second with subsequently ejected, slower moving, material which could consist of thermally ejected C atoms and clusters. We suggest that the second wave forms as the material ejected more slowly collides with the back of the dissipating first shock wave. There is no evidence for this second shock wave being a backwards propagating reflected wave, as its $x$ position increases slowly with time. For fs ablation at a higher $P_{\mathrm{Ar}}$ of 340 mTorr [see Figs. 7(g)-7(i)], the initial shock wave is further retarded compared to that formed at $P_{\mathrm{Ar}}=154$ mTorr and dissipates more quickly, such that by $t=900 \mathrm{~ns}$ only the second compression wave is visible.

A schematic diagram following the evolution of the $\mathrm{C}^{+*}$ emission distribution from the plume is shown in Fig. 8. The scheme shows a cross section though the plume. In this explanation, the existence of a prompt and a delayed source of ejected material is postulated. The first source, with angular

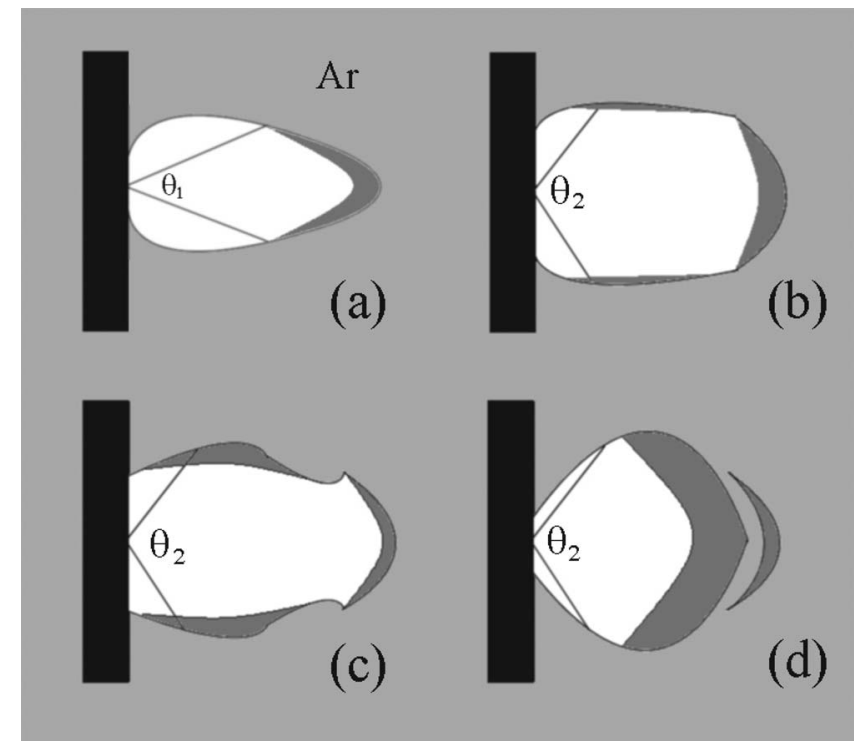

FIG. 8. A schematic diagram following the evolution of the $\mathrm{C}^{+*}$ emission distribution from the plume. The scheme shows a cross section through the plume. In this explanation, the existence of two temporally separated sources is postulated. The first source with angular distribution of $\theta_{1}$ is followed by slower component with angular distribution of $\theta_{2}$.

distribution of $\theta_{1}$, producing a tightly confined shock front [Fig. 8(a)]. Following this initial burst of ablated material is a slower component, with angular distribution of $\theta_{2}$ [see Fig. 8(b)]. Impact ionization of this component by collisions with the gas atoms at the side of the plume produces emission. Later in the evolution [see Fig. 8(c)], the emission from the front shock wave starts to dissipate. The regions of emission at the sides of the plume appear to start to move in behind the shock front. Lastly, in this sequence [Fig. 8(d)] the optical emission from the first shock wave has almost gone and the profile is dominated by emission from the material colliding with the high pressure region at the rear of the first shock front.

After examining the evolution of the $\mathrm{C}^{+*}$ emission, we now discuss the dynamics of the $\mathrm{C}_{2}^{*}$ radical emission for $\mathrm{fs}$ ablation. This was achieved by passing the plume emission through an interference filter with its pass band centered at $525 \mathrm{~nm}$ with a full width at half-maximum of $10 \mathrm{~nm}$. This filter will primarily transmit the emission from $\mathrm{C}_{2}^{*}$ radicals in their $d^{3} \Pi_{u}$ state (and most noticeably, the origin of the $d$-a Swan band system at $516.5 \mathrm{~nm}$ ). There will also be very slight contamination from the $\mathrm{C}^{+*}$ transition at $514.6 \mathrm{~nm}$. Figure 9 shows a series of time-gated CCD images of emission from the fs ablation plume of graphite at different values of $P_{\text {Ar. }}$. Figures 9(a)-9(c) are for ablation in vacuum and are taken at delays of 120, 420, and $900 \mathrm{~ns}$ after the laser pulse, respectively. The peak in the $\mathrm{C}_{2}^{*}$ emission is observed to remain located at the target, as was observed here in the spatially resolved OES measurements and elsewhere. ${ }^{17}$ Lifetime arguments exclude radiative cascade as a mechanism for the persistence of the $C_{2}^{*}$ emission, so another source of $C_{2}^{*}$ is required. Monchicourt, ${ }^{31}$ for $532 \mathrm{~nm}$ nanosecond PLA of graphite, proposed binary collisions between fast and slow $\mathrm{C}$ 


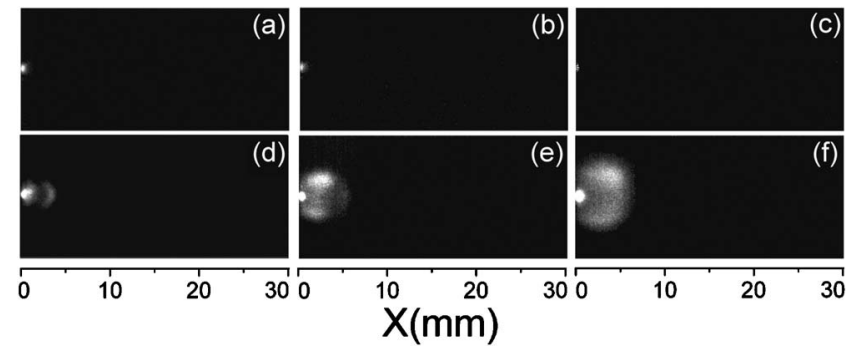

FIG. 9. Series of time-gated CCD images of emission from the fs ablation plume of graphite in different values of $P_{\mathrm{Ar}}$. All the images were taken using a filter that passes the $C_{2}^{*}$ emission. (a)-(c) are for ablation in vacuum and are taken at delays of 120, 420 and $900 \mathrm{~ns}$ after the laser pulse, respectively. (d)-(f) show images obtained at $P_{\mathrm{Ar}}=154 \mathrm{mTorr}$ for the same set of delays.

atoms as a source for $\mathrm{C}_{2}^{*}$, however it is more likely that a third body would be required to stabilize the resultant molecule. Three body collisions are a likely source here as, for ablation in vacuum, collisional processes will occur predominately at the high pressure region near the target. Monchicourt also concludes that binary collisions of $\mathrm{C}$ atoms in excited states would not lead to $\mathrm{C}_{2}^{*}$ production as the internal energy of the initial system would be too large to form a stable molecule.

For $1.06 \mu \mathrm{m}$ Nd:YAG PLA of graphite in He, Sasaki et $a l .{ }^{30}$ monitored the spatial distribution of $\mathrm{C}_{2}$ and $\mathrm{C}_{3}$ radicals by laser induced fluorescence. They concluded that the number of $\mathrm{C}_{2}$ radicals produced by gas phase reactions was comparable with the direct ablation from the target. Here, we expect to have contributions to the $\mathrm{C}_{2}^{*}$ emission from both collisional excitation of $\mathrm{C}_{2}$ and from direct formation of $\mathrm{C}_{2}^{*}$ by binary collisions of $\mathrm{C}$ atoms and three-body interactions between $\mathrm{C}$ atoms and inert gas atoms.

Figures 9(d)-9(f) show images obtained at $P_{\text {Ar }}$ $=154$ mTorr for the same delays after the laser pulse as for ablation in vacuum. At $t=120 \mathrm{~ns}$ [Fig. 9(d)] the emission is still mainly located at the target. However, in addition, a weak emission is observed from the same region as the shock wave seen in the $\mathrm{C}^{+*}$ filtered images. This is either due to contamination of the signal by the $\mathrm{C}^{+*}$ emission, or production of/excitation to $\mathrm{C}_{2}^{*}$ by collisions, or a combination of both effects. At $t=420 \mathrm{~ns}$ [Fig. 9(e)] the emission from the shock front has reduced in intensity dramatically and the peak in the emission is observed at the edges of the region through which the shock wave has propagated. This again suggests that this central region is at a relatively low pressure, the collisions forming the $\mathrm{C}_{2}^{*}$ only occurring at the boundaries. As the initial shock wave observed in the $\mathrm{C}^{+*}$ emission dissipates, the $\mathrm{C}_{2}^{*}$ emission distribution takes the form of second arc-shaped shock wave that propagates slowly away from the target. This second shock wave is in the same position as that observed in the $\mathrm{C}^{+*}$ filtered images. Again, we propose that this second wave of emission occurs as material ejected later, and/or with a slower velocity, collides with the material at the rear of the dissipating first shock wave. The higher intensity of the emissions from the side of the plume, compared with that at the shock front (which is still visible in the $\mathrm{C}^{+*}$ filtered images), indicates
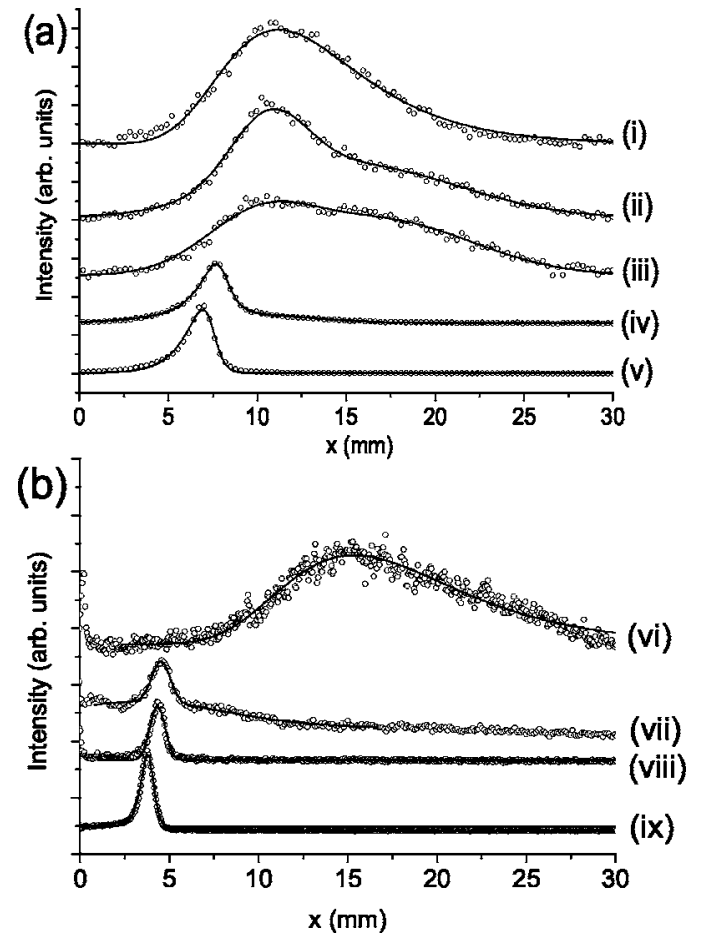

FIG. 10. Intensity $I$ versus $x$ profiles obtained from time-gated CCD images filtered at $426.7 \mathrm{~nm}$ and taken $300 \mathrm{~ns}$ after the laser pulse for (a) ns ablation and (b) femtosecond pulses at different $P_{\mathrm{Ar}}$ values of (i) vacuum, (ii) 5 mTorr, (iii) 20 mTorr, (iv) 68 mTorr, (v) 154 mTorr, (vi) vacuum, (vii) 100 mTorr, (viii) 154 mTorr, and (ix) 340 mTorr.

that collisional excitation of slowly ejected $C_{2}$ may be the dominant source of the $\mathrm{C}_{2}^{*}$ emission.

As previously, ${ }^{27}$ estimates of the apparent mean velocities $\langle v\rangle$ of the imaged species, for both ns and fs ablation, were derived by analyzing the emission intensity profiles along the $x$ axis of each image. Figure 10(a) shows several such profiles, for ns ablation, and their variation with $P_{\mathrm{Ar}}$, for data recorded at $t=300 \mathrm{~ns}$. These plots illustrate the bimodal nature of the emission distribution at low and intermediate $P_{\mathrm{Ar}}$ values (an observation that will be discussed more fully later, and appears more dramatically, when the ablation in He is investigated). Forward peaking of the distributions measured at high $P_{\mathrm{Ar}}$ is also evident. A similar set of profiles for the ablation using fs pulses, also measured $t=300 \mathrm{~ns}$ after the laser pulse, are shown in Fig. 10(b). In vacuum, the profile for fs pulses is similar to that for ns ablation, but the distribution is broader and further advanced. At the higher values of $P_{\mathrm{Ar}}$, the profiles across the shock wave, for fs pulses, are much narrower and more retarded when compared to ns ablation. The plume confinement appears more significant for the initial shock wave formed by fs ablation. This suggests that the high energy impacting species forming the initial shock wave are emitted over a shorter period of time than for fs pulses.

The peak (or peaks) and the center of gravity of each distribution were found by fitting with suitable mathematical functions, the exact nature of which varied, to allow systematic fitting of the plume evolution. Figure 11(a) shows how 


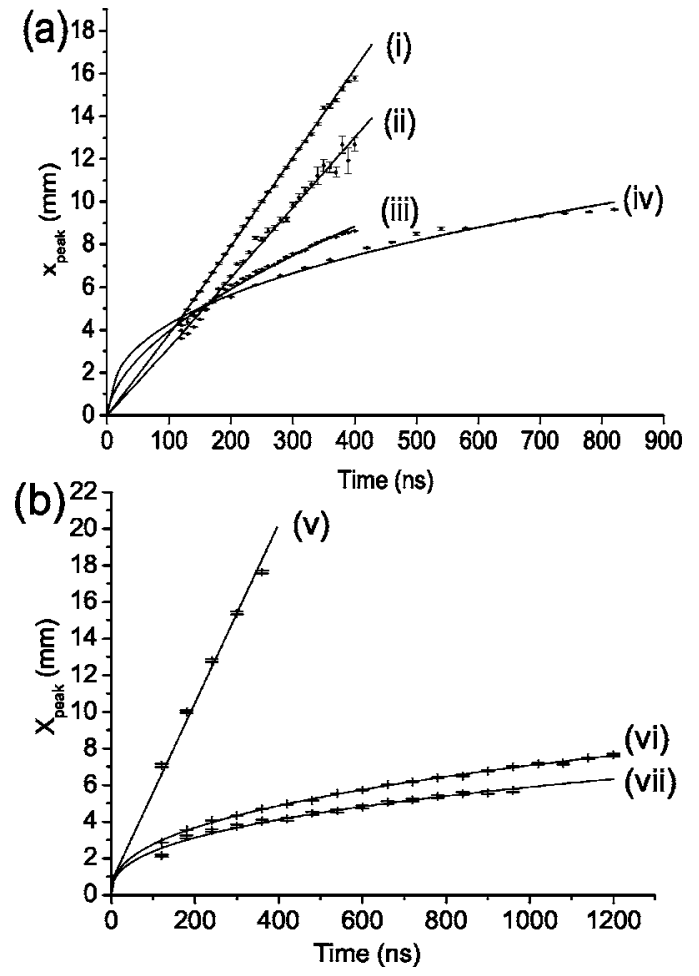

FIG. 11. Plots of $x_{\text {peak }}$ versus time for the $\mathrm{C}^{+}$emissions following (a) nanosecond and (b) femtosecond PLA of graphite in background pressures of $P_{\mathrm{Ar}}=$ (i) vacuum (ii) $20 \mathrm{mTorr}$, (iii) $68 \mathrm{mTorr}$, (iv) 154 mTorr, (v) vacuum, (vi) 154 mTorr, and (vii) 340 mTorr. The solid lines are fits to the data in terms of Eq. (2) with values $\alpha=$ (i) 1.00 , (ii) 1.00 , (iii) 0.58 , (iv) 0.40 , (v) 1.00 , (vi) 0.40 , and (vii) 0.40 .

the derived peak in the profiles $x_{\text {peak }}$ varies with $t$, as a function of $P_{\mathrm{Ar}}$, for ns pulses. Figures 11 illustrates the good linear relationship between $x_{\text {peak }}$ and time $t$ observed for $248 \mathrm{~nm}$ PLA of graphite, in vacuum. The gradient of this plot gives a propagation velocity $\langle v\rangle$ of $41.5 \pm 0.1 \mathrm{~km} / \mathrm{s}$ for the $\mathrm{C}^{2+}$ species (remembering that we asserted earlier that in vacuum the $\mathrm{C}^{+*}$ emission comes from EIR of $\mathrm{C}^{2+}$ ). This is equivalent to a $\mathrm{C}$ atom kinetic energy of $\sim 107 \mathrm{eV}$, and is in excellent accord with values obtained previously for such species formed in the PLA of graphite in vacuum, using nanosecond $193 \mathrm{~nm}$ and $248 \mathrm{~nm}$ laser pulses and comparable incident fluences. ${ }^{17,27} a$-C films grown from ablation plumes containing $\mathrm{C}$ cations with energies in this range typically tend to have a high $(\sim 80 \%)$ fraction of $s p^{3}$ bonding. ${ }^{21}$

As stated earlier, the filtered images of the evolving plume in 20 mTorr of argon show a bimodal velocity distribution. This bimodality has the effect of making all $x_{\text {peak }}$ versus $t$ plots obtained at $P_{\mathrm{Ar}}>0$ nonlinear, with a curvature that increases with $P_{\mathrm{Ar}}$. This curvature is not noticeable at $P_{\mathrm{Ar}}=20 \mathrm{mTorr}$ and the $\langle v\rangle$ extracted is $36.0 \pm 0.4 \mathrm{~km} / \mathrm{s}$. Such bimodality, or plume splitting, has been reported previously both for ions and neutral species formed by PLA in low pressures of background gas. $1,8,37$ In the present case, the observed bimodal distribution most probably reflects contributions to the observed $\mathrm{C}^{+*}$ emission both from EIR involving $\mathrm{C}^{2+}$ ions (as for PLA in vacuum) and from collision induced impact ionization.
Collisional contributions would be expected to increase, and the apparent propagation velocity to slow, with increasing $P_{\mathrm{Ar}}$. Consistent with such expectations, we see that for $P_{\text {Ar }}=68$ mTorr [see Fig. 11(a), iii], the relationship between $x_{\text {peak }}$ and $t$ has a marked negative curvature: the extracted velocity (obtained from tangents to the curve) reduces from $33 \pm 1.1 \mathrm{~km} / \mathrm{s}$ at $t=120 \mathrm{~ns}$ to $8.2 \pm 0.4 \mathrm{~km} / \mathrm{s}$ at $400 \mathrm{~ns}$. Such effects are even more marked for the data recorded at $P_{\text {Ar }}=154$ mTorr, for which the tangents to the best-fit curve imply an even more dramatic deceleration, from $17.7 \pm 0.4 \mathrm{~km} / \mathrm{s}$ at $120 \mathrm{~ns}$ to just $8.0 \pm 0.4 \mathrm{~km} / \mathrm{s}$ at $400 \mathrm{~ns}$ and $2.6 \pm 0.1 \mathrm{~km} / \mathrm{s}$ at $800 \mathrm{~ns}$. Figure 11(b) shows a similar set of $x_{\text {peak }}$ versus $t$ plots for fs ablation of graphite in Ar. In vacuum [fig. 11(b), v] a good linear relationship between $x_{\text {peak }}$ and time $t$ is observed again, giving $\langle v\rangle=48.9 \pm 0.1 \mathrm{~km} / \mathrm{s}$. This value is significantly higher than for ns pulses, a typical observation when comparing fs and ns ablation. ${ }^{17,29}$ At higher pressures, a similar apparent deceleration of the emission peak is observed.

Amoruso et al. ${ }^{37}$ have reported qualitatively similar trends in the case of $\mathrm{Mg}^{*}$ atoms formed in the $351 \mathrm{~nm}$ PLA of an $\mathrm{MgB}_{2}$ target in low background pressures of Ar. As here, they deduce an increasingly intense plume-background interaction at increasing $P_{\text {Ar }}$, leading to a shock-wave-like expansion, plume confinement, and plume heating (to an extent not found in free plume expansion into vacuum). This, in turn, leads to enhanced emission from the plume core and enhanced excitation/ionization at the plume front where the high collision rate encourages conversion of the plume stream velocity into thermal energy. ${ }^{33,37}$ As in the case of $\mathrm{Mg}^{*}$ atoms from PLA of $\mathrm{MgB}_{2}$, we find that the data sets obtained at $P_{\mathrm{Ar}}>0$, for both ns and fs pulses, are found to be reproduced well by a simple function of the form

$$
x_{\text {peak }} \propto t^{\alpha}
$$

(see Fig. 11). Such an expression was introduced to model the expansion of a spherical blast-wave ${ }^{38}$ - wherein the exponent $\alpha$ takes a limiting value of $\sim 0.4-$ but, as in the $\mathrm{MgB}_{2}$ study, ${ }^{37}$ we find it to provide a very adequate description of the time dependent propagation of the $\mathrm{C}^{+*}$ emission from the shock wave produced by both ns and fs pulses.

After examining the ablation in $P_{\text {Ar }}$, we briefly discuss the plume dynamics for fs ablation of graphite in $P_{\mathrm{He}}$. For light carbon atoms in a heavy Ar background, we can predict with simple mechanics, that there will be significant backscattering of $\mathrm{C}$ atoms. For head-on collisions with He atoms, however, which are lighter than $\mathrm{C}$, we would expect no backscattering. This fact, along with the smaller collisional cross section for He means that a higher $P_{\mathrm{He}}$, compared to $\mathrm{Ar}$, is required to have a comparable effect on the plume dynamics. A review of the dynamics of plume propagation and splitting for $\mathrm{Si}$ ns ablation in $\mathrm{Ar}$ and $\mathrm{He}$ is given by Wood et al. ${ }^{8}$

Figure 12 shows a series of wavelength filtered CCD images of the fs ablation plume for $P_{\mathrm{He}}=4$ Torr. Images (a)-(c) are filtered to pass the $\mathrm{C}^{+*}$ emission and were taken with a variable delay after the laser pulse of $120 \mathrm{~ns}$ for the images in the first column, $420 \mathrm{~ns}$ for the second column and $900 \mathrm{~ns}$ for the third column. At $t=120 \mathrm{~ns}$, two distinct regions of $\mathrm{C}^{+*}$ emission are observed. One broad, fast component and 


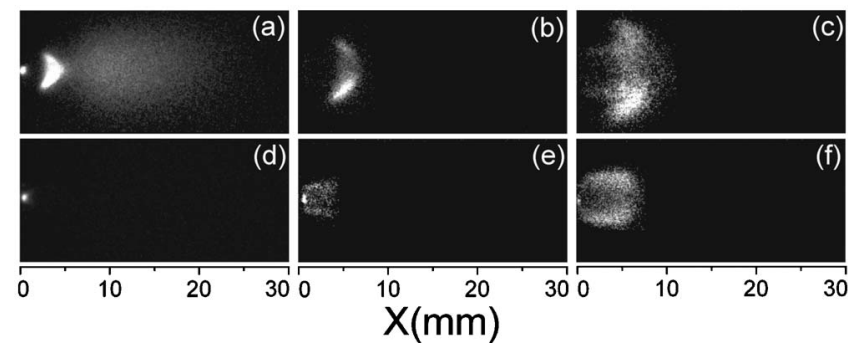

FIG. 12. Wavelength filtered CCD images of the fs ablation plume for $P_{\mathrm{He}}=4$ Torr. Images (a)-(c) are filtered to pass the $\mathrm{C}^{+*}$ emission and (d)-(f) to pass the $\mathrm{C}_{2}^{*}$ radical emission. Images were taken with a variable delay after the laser pulse of $120 \mathrm{~ns}$ for images in the first column, $420 \mathrm{~ns}$ for the second column and $900 \mathrm{~ns}$ for the third column.

another confined, "arrow-head" shaped shock front. By 420 ns and beyond, the peak of the fast component has moved out of the viewing region. The remaining shock front decelerates into the background gas, in a similar manner to the ablation in Ar, but a gap at the point of the "arrow head" is observed which may be due to the fast component "sweeping away" the background gas in the forward direction. Emission is also observed at the edges of the lower pressure region behind the shock front. In addition to examining the $\mathrm{C}^{+*}$ emission, the images in Figs. 12(d)-12(f) were taken using the filter which passes the $\mathrm{C}_{2}^{*}$ radical emission. The evolution of this distribution is very similar to that observed for the fs ablation of graphite in Ar, the emission mainly coming from the edges of the vacuum space produced by the passing initial shock front. At times later than $900 \mathrm{~ns}$, this emission distribution changes shape into an arc of emission propagating away from the target.

In the same manner as before, the apparent velocity of propagation of the plume components can be extracted by analyzing the profiles along the $x$-axis. One such profile, from the $\mathrm{C}^{+*}$ filtered image at $t=120 \mathrm{~ns}$, is shown in Fig. 13(a). This profile clearly demonstrates a bimodal distribution in the emission. By fitting the positions of these two peaks, and plotting them against the time [see Fig. 13(b)] we extract a propagation $\langle v\rangle$ of $83.3 \pm 0.1 \mathrm{~km} / \mathrm{s}$ for the fast peak and a decelerating shock wave propagation for the slow component. The $\langle v\rangle$ of the fast component is much higher than the velocity for $\mathrm{C}^{2+}$ ions extracted from the ablation in vacuum. It is suggested that this front component is due to high energy collisions, of the faster moving components in the ablation plume (which haven't suffered any collisions yet), with $\mathrm{He}$ atoms.

The rate of decrease in the deduced velocity with increasing background pressure of inert gas $\left(P_{\text {inert }}\right)$ and/or $t$ might suggest that, at the higher pressures studied, the ablated material would not reach the substrate. This is not necessarily the case, however. It is probably worth reiterating that the present experiments are sensitive only to that fraction of the plume material that is excited to the particular emitting states under investigation. The peak in the spatial distribution of $\mathrm{C}^{+*}$ ions at high $\mathrm{P}_{\text {inert }}$ is likely to be near the position of highest plume density and thus highest collisional excitation/ ionization probability. The velocity we derive at high $P_{\text {inert }}$ is

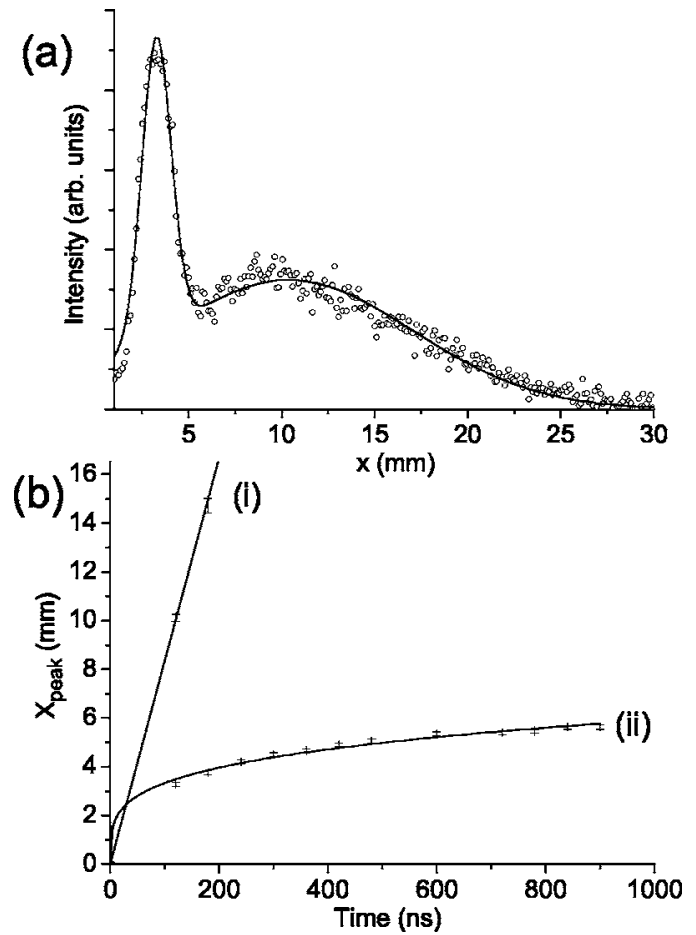

FIG. 13. (a) Intensity $I$ versus $x$ profile obtained from time-gated CCD image of the fs ablation plume, filtered at $426.7 \mathrm{~nm}$ and taken 120 ns after the laser pulse, for $P_{\mathrm{He}}=4$ Torr, (b) plots of $x_{\text {peak }}$ versus time for the $\mathrm{C}^{+}$emissions following (i) the "fast" and (ii) "slow" peaks that are evidenced in (a).

thus the propagation velocity of the shock wave formed as the plume expands into the background gas. After impact excitation, and subsequent radiative decay, the (now dark) species in the plume will propagate away from the shockfront with some (at least partially thermalized) velocity. Clearly, the present experiments are blind to the substantial amount of "dark" ablated material, some of which at least will reach the substrate without suffering collisional excitation and will thus make no contribution to the measured velocity.

Before going on to discuss the formation of $\mathrm{C}$ clusters in the plume, we summarize the results of the plume imaging experiments.

(i) For ablation in vacuum the velocity of $\mathrm{C}^{2+}$ ions in the plume can be extracted from series of images of $\mathrm{C}^{+*}$ emission. The value obtained for fs ablation is significantly larger than that for ns pulses.

(ii) In plumes confined by ambient gas, the impact ionization/excitation of $\mathrm{C}$ species dominates the optical emission and can be used to track the position of the shock wave formed as the plume expands into the background gas.

(iii) For ns pulses the propagation of the shock wave is reproduced well by a spherical blast-wave model. For fs pulses however, the plume dynamics are more complex. A primary shock wave is produced by an initial burst of highly energetic ablated $\mathrm{C}$ species. As the primary shock wave dissipates, a second shock wave is formed by the collisions of subsequently ejected, slower moving material. 


\section{Cluster formation in the plume}

Once the highly energetic ablated atomic material has been slowed by collisions, the formation of carbon clusters become a possibility. The size and bonding configuration within these clusters is then controlled by the plume temperature and the density of carbon atoms. ${ }^{11,30,31}$ We now discuss how the initial plume dynamics observed here, for both $\mathrm{ns}$ and fs pulses, affect the subsequent $\mathrm{C}$ cluster formation in the expanding plume.

The element carbon has a rather unique property that it forms clusters at elevated temperatures, such as in flames, astrophysical environments and in laser vaporized carbon plumes. ${ }^{31,39}$ One important consideration regarding the growth of clusters in the plume is the source of the initial cluster seeds, which are likely to be $\mathrm{C}$ clusters with a small number of atoms $(N)$. Three of the main sources for these seeds are likely to be the following: (a) Collisions between $\mathrm{C}$ atoms (stabilized by a third body) as a source for $\mathrm{C}_{2}$ and between $\mathrm{C}_{2}$ and a $\mathrm{C}$ atom for $\mathrm{C}_{3}$. (b) The direct ablation of small $N$ clusters and chains and (c) the collisional fragmentation of larger $N$ clusters into multiple small $N$ clusters. The relative importance of the three suggested sources is likely to be different for the two pulse durations. From the OES measurements for fs ablation in vacuum, the higher relative intensity of $\mathrm{C}^{+*}$ compared to $\mathrm{C}^{*}$ emissions (a trend that is reversed for ns pulses) could imply that the fraction of $\mathrm{C}$ ablated as ground state atoms is less for fs pulses compared with ns pulses. If so, the production of $\mathrm{C}_{2}$ by gas phase reactions would be expected to be less efficient for fs pulses. This cannot be confirmed, however, as OES measurement are blind to the "dark" fraction of the plume. Also from the OES measurements, it appears that fs pulses directly eject more $\mathrm{C}_{2}$ and $\mathrm{C}_{3}$ than for ns ablation. This is either due to the nature of the ablation mechanism, or to photodissociation of $\mathrm{C}$ clusters in the plume by the ns pulses. Simulations of the ultrafast ablation of graphite have also shown that the ablation of fast monomers is accompanied by the production of significant numbers of $\mathrm{C}$ clusters and chains. ${ }^{14}$ The collisional fragmentation of larger clusters is likely to be mainly controlled by the background pressure in the chamber, which will be irrespective of pulse duration. However, the initial production of these larger clusters will be determined by the nature of the initial ablation event.

After formation of these clusters, the dominant growth mechanism is then expected to be single-atom attachment to larger clusters. ${ }^{11,30}$ This process will be limited by the density of $\mathrm{C}$ atoms in the plume. If the density of $\mathrm{C}_{2}$ and $\mathrm{C}_{3}$ species in the plume is high enough, cluster growth by other purely binary collisions such as $\mathrm{C}_{2}+\mathrm{C}_{2} \rightarrow \mathrm{C}_{3}+\mathrm{C}$ and $\mathrm{C}_{2}$ $+\mathrm{C}_{3} \rightarrow \mathrm{C}_{4}+\mathrm{C}$ could also be significant. Rode et al. ${ }^{11}$ postulated that a threshold density of $\mathrm{C}$ atoms in the plume is required for significant cluster formation, and that the characteristic time of formation $\left(t_{N}\right)$ of an cluster with $N$ atoms can be estimated by

$$
t_{N}=\frac{1}{e} N\left(\frac{n_{\mathrm{Ar}}}{n_{1}^{2}} \sigma \nu\right),
$$

where $e$ is the base of the natural logarithm, $n_{1}$ is the $\mathrm{C}$ atom density, $n_{\mathrm{Ar}}$ is the Ar atom density, $\sigma$ is the $\mathrm{C}$-Ar collision cross section, and $\nu$ is the $\mathrm{C}$ atom velocity.
Although the fluences used for the two pulse durations were similar, the ns pulses contained significantly more energy and typically ablate considerably more material per pulse. ${ }^{29}$ To a crude approximation it could be assumed that the total amount of material ablated, per pulse, is proportional to the laser energy (well above the ablation threshold). Of course, here we are ignoring the differences in the ablation mechanisms between fs and ns pulses. If this assumption is valid, the number of $\mathrm{C}$ atoms ablated into in the cluster forming regions of the plume will be smaller for fs pulses by a factor of $\sim 8.5$. If we assume that the size of the cluster forming region (or at least the the region where the initial $\mathrm{C}_{2}$ and $\mathrm{C}_{3}$ cluster seeds are formed) is represented by the volume of the shock wave observed during the plume imaging, the density of carbon atoms for fs ablation would be increased by a factor of $\sim 2$ due to the enhanced plume confinement observed for fs pulses. Thus, if we combine these two factors, the $t_{N}$ for an cluster with $N$ atoms should be $\sim 18$ times longer for the fs ablation compared with ns ablation. If we assume that, for both pulse durations, the plume remains at suitable temperature for cluster formation for similar amounts of time, we would expect much smaller clusters to be produced in the case of fs ablation. This will be irrespective of the initial density of cluster seeds produced by the two pulse durations. Indeed, from the SEM images shown in Fig. 1, there was no evidence for the deposition of carbon nanofoam material when using fs pulses. This may be an indication that the growth of the larger clusters was not occurring. However, analysis of the cluster size in the plume by probe beam scattering experiments should be performed to confirm this hypothesis.

\section{CONCLUSIONS}

Wavelength filtered imaging of the plume emissions accompanying the $248 \mathrm{~nm}$ PLA of graphite with short and ultrashort pulses, in different background pressures of inert gas, was performed. For ablation at low $P_{\mathrm{Ar}}(<20 \mathrm{mTorr})$ the propagation of $\mathrm{C}$ species in the plume appeared to be relatively unaffected by the gas. The deduced kinetic energies of $\mathrm{C}^{2+}$ ions (inferred from the images of $\mathrm{C}^{+*}$ emission) in this regime was $\sim 100 \mathrm{eV}$, a value traditionally associated with the formation of dense, $s p^{3}$ rich, $a$-C films, as was observed. The mean velocity of the plume components was noticeably reduced at higher $P_{\mathrm{Ar}}$, as a result of collisions, the $\mathrm{C}^{+*}$ emissions in this regime were deduced to be dominated by impact ionization and excitation. Shock wave formation, as the plume tried to expand against the background gas, was clearly evident at $P_{\mathrm{Ar}} \sim 68$ mTorr. At $P_{\mathrm{Ar}}>150$ mTorr, the deduced deceleration of the shock wave with increasing $t$ could imply that much if not all of the plume expansion would dissipate before reaching the substrate. Consistent with this view, we observe deposition of low density, filamentary films in this pressure regime, suggesting that the majority growth species are clusters formed in the gas phase, which reach the substrate with energies below that required for coalescence into the bulk of the film to occur. This suggestion is supported by the TEM images of the samples that appear to show that these films grow by diffusion limited aggregation of nanoscale carbon clusters. 
For ablation with fs pulses, the propagation of two shock waves was observed. It is suggested that the first is associated with an initial burst of highly energetic/electronically excited ablated components, indicative of the enhanced fraction of nonthermal ejection mechanisms compared to ns ablation, and the second with subsequently ejected, slower moving, material which could consist of thermally ejected C atoms and clusters. Supporting this assignment, the presence of a lower pressure gap, behind the first shock front, is observed for fs pulses. For ns ablation, the plume components are expected to have a more continuous spread of velocities as the source of "suprathermal" ions is likely to be absorption of the laser radiation (e.g., by inverse Brehmsstrahlung excitation) by the expanding thermally generated plume, leading to heating, excitation and ionization of the plume constituents.

The enhanced confinement of the initial shock wave and the suggested increase in production of $\mathrm{C}$ clusters, for $\mathrm{fs}$ pulses, could be beneficial for the growth of $\mathrm{C}$ nanoclusters, but the significantly smaller volume of material ablated per pulse leads to a much lower $\mathrm{C}$ atom density in the plume, which limits the growth of clusters. One method to overcome this drawback would be to use high-repetition-rate laser ablation, where the time between successive pulses is less than the dwell time of the carbon atoms in the plume. In this regime the $\mathrm{C}$ atom density can be "pumped" and sustained at cluster forming levels. The reported plume energetics can be applied to ablation in many material system and also has applicability for cathodic arc deposition under background pressures.

\section{ACKNOWLEDGMENTS}

The authors thank Professor Costas Fotakis for his assistance with this project and S.J.H. and G.M.F. would like to thank A. Egglezis and A. Klini for helping with the laser and vacuum systems, respectively. The authors are grateful to EPSRC for funding via two portfolio grants LASER and Integrated Electronics and the Carbon Based Electronics programme. Experiments were carried out at the Ultraviolet Laser Facility operating at IESL FORTH and supported by the EU through the Research Infrastructures activity of FP6 (Project: Laserlab-Europe Contract No. RII3-CT-2003506350).
*Electronic address: s.henley@eim.surrey.ac.uk

${ }^{1}$ R. F. Wood, K. R. Chen, J. N. Leboeuf, A. A. Puretzky, and D. B. Geohegan, Phys. Rev. Lett. 79, 1571 (1997).

${ }^{2}$ M. N. R. Ashfold, F. Claeyssens, G. Fuge, and S. J. Henley, Chem. Soc. Rev. 33, 23 (2004), and references therein.

${ }^{3}$ R. K. Thareja and R. K. Dwivedi, Phys. Lett. A 222, 199 (1996).

${ }^{4}$ M. P. Siegal, D. L. Overmyer, R. J. Kottenstette, D. R. Tallant, and W. G. Yelton, Appl. Phys. Lett. 80, 3940 (2002).

${ }^{5}$ A. A. Voevodin, S. J. P. Laube, S. D. Walck, J. S. Solomon, M. S. Donley, and J. S. Zabinski, J. Appl. Phys. 78, 4123 (1995).

${ }^{6}$ H. C. Ong and R. P. H. Chang, Phys. Rev. B 55, 13213 (1997).

${ }^{7}$ Y. Lifshitz, S. R. Kasi, and J. W. Rabalais, Phys. Rev. Lett. 62, 1290 (1989).

${ }^{8}$ R. F. Wood, J. N. Leboeuf, D. B. Geohegan, A. A. Puretzky, and K. R. Chen, Phys. Rev. B 58, 1533 (1998).

${ }^{9}$ Z. Geretovszky, T. Haraszti, T. Szörényi, F. Antoni, and E. Fogarassy, Appl. Surf. Sci. 208-209, 566 (2003).

${ }^{10}$ B. S. Satyanarayana, J. Robertson, and W. I. Milne, J. Appl. Phys. 87, 3126 (2000).

${ }^{11}$ A. V. Rode, E. G. Gamaly, and B. Luther-Davies, Appl. Phys. A: Mater. Sci. Process. 70, 135 (2000).

${ }^{12}$ R. F. Service, Science 304, 42 (2004).

${ }^{13}$ S. Amoruso, X. Wang, C. Altucci, C. de Lisio, M. Armenante, R. Bruzzese, and R. Velotta, Appl. Phys. Lett. 77, 3728 (2000).

${ }^{14}$ H. O. Jeschke, M. E. Garcia, and K. H. Bennemann, Phys. Rev. Lett. 87, 015003 (2001).

${ }^{15}$ H. Koster and K. Mann, Appl. Surf. Sci. 110, 428 (1997).

${ }^{16}$ S. I. Anisimov, B. Kapeliovich, and T. Perel'man, JETP 39, 375 (1994).

${ }^{17}$ F. Claeyssens, M. N. R. Ashfold, E. Sofoulakis, C. G. Ristoscu, D. Anglos, and C. Fotakis, J. Appl. Phys. 91, 6162 (2002).

${ }^{18}$ S. Szatmari and P. F. Schafer, Opt. Commun. 68, 196 (1988).

${ }^{19}$ S. Tzortzakis, B. Lamouroux, A. Chiron, S. D. Moustaizis, D.
Anglos, M. Franco, B. Prade, and A. Mysyrowicz, Opt. Commun. 197, 131 (2001).

${ }^{20}$ T. A. Witten and L. M. Sander, Phys. Rev. Lett. 47, 1400 (1981).

${ }^{21}$ A. A. Voevodin and M. S. Donley, Surf. Coat. Technol. 82, 199 (1996).

${ }^{22}$ S. J. Henley, J. D. Carey, and S. R. P. Silva, Appl. Phys. Lett. 85, 6236 (2004).

${ }^{23}$ M. A. Tamor and W. C. Vassel, J. Appl. Phys. 76, 3823 (1994).

${ }^{24}$ A. C. Ferrari and J. Robertson, Phys. Rev. B 61, 14095 (2000).

${ }^{25}$ J. Schwan, S. Ulrich, V. Batori, H. Ehrhardt, and S. R. P. Silva, J. Appl. Phys. 80, 440 (1996).

${ }^{26}$ E. Barborini, P. Piseri, A. L. Bassi, A. C. Ferrari, C. E. Bottani, and P. Milani, Chem. Phys. Lett. 300, 633 (1999).

${ }^{27}$ F. Claeyssens, R. J. Lade, K. N. Rosser, and M. N. R. Ashfold, J. Appl. Phys. 89, 697 (2001).

${ }^{28}$ S. Acquaviva and M. L. De Giorgi, Appl. Surf. Sci. 197, 21 (2002).

${ }^{29}$ S. J. Henley, G. M. Fuge, and M. N. R. Ashfold, J. Appl. Phys. 97, 023304 (2005).

${ }^{30}$ K. Sasaki, T. Wakasaki, S. Matsui, and K. Kadota, J. Appl. Phys. 91, 4033 (2002).

${ }^{31}$ P. Monchicourt, Phys. Rev. Lett. 66, 1430 (1991).

${ }^{32}$ NIST Atomic Spectra Database, http://physics.nist.gov/cgi-bin/ AtData/main_asd

${ }^{33}$ N. Arnold, J. Gruber, and J. Heitz, Appl. Phys. A: Mater. Sci. Process. 69, S87 (1999).

${ }^{34}$ I. Zerogioti, S. Mailis, C. Fotakis, S. C. Chen, and C. P. Grigoropoulos, Appl. Surf. Sci. 126, 601 (1998).

${ }^{35}$ A. S. Loir, F. Garrelie, J. L. Subtil, F. Goutaland, M. Belin, R. Le Harzic, C. Donnet, Y. Ouerdane, F. Rogemond, and P. Laporte, Appl. Surf. Sci. 208, 553 (2003).

${ }^{36}$ S. Amoruso, G. Ausanio, M. Vitiello, and X. Wang, Appl. Phys. A: Mater. Sci. Process. 81, 981 (2005). 
${ }^{37}$ S. Amoruso, B. Toftmann, J. Schou, R. Velotta, and X. Wang, Thin Solid Films 453-454, 562 (2004).

${ }^{38}$ Y. B. Zel'dovich and Y. P. Raizer, Physics of Shock Waves and High-Temperature Hydrodynamic Phenomena (Academic Press,
New York, 1966).

${ }^{39}$ W. Weltner and R. J. Van Zee, Chem. Rev. (Washington, D.C.) 89, 1713 (1989). 